\title{
M-FUNCTIONS AND INVERSE SPECTRAL ANALYSIS FOR FINITE AND SEMI-INFINITE JACOBI MATRICES
}

\author{
Fritz Gesztesy ${ }^{1}$ AND BARRY SIMON ${ }^{2}$
}

\begin{abstract}
We study inverse spectral analysis for finite and semi-infinite Jacobi matrices $H$. Our results include a new proof of the central result of the inverse theory (that the spectral measure determines $H$ ). We prove an extension of Hochstadt's theorem (who proved the result in the case $n=N$ ) that $n$ eigenvalues of an $N \times N$ Jacobi matrix, $H$, can replace the first $n$ matrix elements in determining $H$ uniquely. We completely solve the inverse problem for $\left(\delta_{n},(H-z)^{-1} \delta_{n}\right)$ in the $N<\infty$ case.
\end{abstract}

\section{$\S 1$. Introduction}

There is an enormous literature on inverse spectral problems for $-\frac{d^{2}}{d x^{2}}+V(x)$ (see $[1$, $29,56-60,64]$ and references therein), but considerably less for their discrete analog, the infinite and semi-infinite Jacobi matrices (see, e.g., [3, 4, 6-8, 13-22, 24, 26-28, 30, 32, $37,38,42-44,50-52,61-63,66,67,69-71]$ ) and even less for finite Jacobi matrices (where references include, e.g., $[9,10,23,25,39,40,41,45-48])$. Our goal in this paper is to study the last two problems using one of the most powerful tools from the spectral theory of $-\frac{d^{2}}{d x^{2}}+V(x)$, the $m$-functions of Weyl.

Explicitly, we will study finite $N \times N$ matrices of the form:

$$
H=\left(\begin{array}{ccccccc}
b_{1} & a_{1} & 0 & 0 & \cdot & \cdot & \cdot \\
a_{1} & b_{2} & a_{2} & 0 & \cdot & \cdot & \cdot \\
0 & a_{2} & b_{3} & a_{3} & \cdot & \cdot & \cdot \\
\cdot & \cdot & \cdot & \cdot & \cdot & \cdot & \cdot \\
\cdot & \cdot & \cdot & \cdot & \cdot & \cdot & \cdot \\
\cdot & \cdot & \cdot & \cdot & 0 & a_{N-1} & b_{N}
\end{array}\right)
$$

and the semi-infinite analog $H$ defined on

$$
\ell^{2}(\mathrm{~N})=\left\{u=\left.(u(1), u(2), \ldots)\left|\sum_{n=1}^{\infty}\right| u(n)\right|^{2}<\infty\right\}
$$

\footnotetext{
${ }^{1}$ Department of Mathematics, University of Missouri, Columbia, MO 65211, USA. E-mail: mathfg@mizzou1.missour

2 Division of Physics, Mathematics, and Astronomy, California Institute of Technology, Pasadena, CA 91125, USA. E-mail: bsimon@caltech.edu

This material is based upon work supported by the National Science Foundation under Grant Nos. DMS9623121 and DMS-9401491.
} 
given by

$$
\begin{aligned}
(H u)(n) & =a_{n} u(n+1)+b_{n} u(n)+a_{n-1} u(n-1), & & n \geq 2 \\
& =a_{1} u(2)+b_{1} u(1), & & n=1 .
\end{aligned}
$$

In both cases, the $a$ 's and $b$ 's are real numbers with $a_{n}>0$.

To avoid inessential technical complications, we will only consider the case where $\sup _{n}\left[\left|a_{n}\right|+\square\right.$ $\left.\left|b_{n}\right|\right]<\infty$ in which case $H$ is a map from $\ell^{2}$ to $\ell^{2}$ and defines a bounded self-adjoint operator.

In the semi-infinite case, we will set $N=\infty$. At times to have unified notation, we will use something like $1 \leq j<N+1$ to indicate $1 \leq j \leq N$ in the finite case and $1 \leq j<\infty$ in the semi-infinite case.

It will sometimes be useful to consider the $b$ 's and $a$ 's as a single sequence $b_{1}, a_{1}, b_{2}, a_{2}, \cdots:=$ $c_{1}, c_{2}, \ldots$, that is,

$$
c_{2 n-1}=b_{n}, \quad c_{2 n}=a_{n}, \quad n=1,2, \ldots
$$

What makes Jacobi matrices special among all matrices is that the eigenvalue condition $H u=\lambda u$ is a second-order difference equation. The $n=1$ case of (1.2) can be thought of as forcing the Dirichlet boundary condition $u(0)=0$. Thus, any possible non-zero solution of $H u=\lambda u$ must have $u(1) \neq 0$, which implies

(i) Eigenvalues of $H$ must be simple (or else a linear combination would vanish at $n=1)$.

(ii) Eigenfunctions must be non-vanishing at $n=1$.

Thus for $N<\infty, H$ has eigenvalues $\lambda_{1}<\cdots<\lambda_{N}$ and associated orthonormal eigenvectors $\varphi_{1}, \ldots, \varphi_{N}$ with $\varphi_{j}(1) \neq 0$. For $N=\infty$, the proper way of encompassing (i), (ii) is that $\delta_{1}$ is a cyclic vector for $H\left(\delta_{j}\right.$ is the vector in $\ell^{2}$ with $\delta_{j}(n)=1$ (resp. 0) if $n=j$ (resp. $n \neq j$ )).

The spectral measure $d \rho$ for the pair $\left(H, \delta_{1}\right)$ is defined by $\left(\delta_{1}, H^{\ell} \delta_{1}\right)=\int \lambda^{\ell} d \rho(\lambda)$. Since our $H$ 's are bounded, $d \rho$ is a measure of bounded support. In case $N<\infty$,

$$
d \rho(\lambda)=\sum_{j=1}^{N}\left|\varphi_{j}(1)\right|^{2} \delta\left(\lambda-\lambda_{j}\right) d \lambda \quad\left(\varphi_{j}, \varphi_{k}\right)=\delta_{j, k} .
$$

The central fact of the inverse theory is that $d \rho$ determines the $a$ 's and $b$ 's and any $d \rho$ can occur for a unique $H$. (If $N<\infty, d \rho$ has support at exactly $N$ points. If $N=\infty$, $d \rho$ must have infinite support.) The usual proof of this central fact is via orthogonal polynomials, and has been rediscovered by many people (see references in the appendix). For the reader's convenience, we have a brief appendix presenting this approach.

One purpose of this paper is to present in Section 3 a new approach to the central result based on $m$-functions and trace formulas. Given $\rho$, one forms $m(z)=\int d \rho(\lambda)(\lambda-z)^{-1}$. $m(z)$ has an asymptotic expansion at infinity given by

$$
m(z) \sim-\frac{1}{z}-\frac{b_{1}}{z^{2}}-\frac{a_{1}^{2}+b_{1}^{2}}{z^{3}}+O\left(z^{-4}\right) .
$$

Thus, one easily recovers $b_{1}$ and $a_{1}$ (recall $a_{1}>0$ ) from $m(z)$. Now define $m_{1}(z)$ by

$$
(-m(z))^{-1}=z-b_{1}+a_{1}^{2} m_{1}(z) .
$$


It turns out that $m_{1}(z)$ is the spectral measure for the Jacobi matrix obtained by removing the top row and left-most column of $H$. An obvious inductive procedure obtains $b_{2}, a_{2}, \ldots$

The $m$-functions defined by this method, which we call $m_{+}(z, n)\left(\right.$ so $m(z):=m_{+}(z, 0)$, $m_{1}(z):=m_{+}(z, 1)$, etc. $)$, is one class of $m$-functions defined by

$$
m_{+}(z, n)=\left(\delta_{n+1},\left(H_{[n+1, N]}-z\right)^{-1} \delta_{n+1}\right),
$$

where $H_{[n+1, N]}$ is the matrix with the top $n$ rows and left $n$ columns removed and thought of as acting on $\ell^{2}(\{n+1, n+2, \ldots, N\})$. There is a second $m$-function that plays a role,

$$
m_{-}(z, n)=\left(\delta_{n-1},\left(H_{[1, n-1]}-z\right)^{-1} \delta_{n-1}\right)
$$

where $H_{[1, n]}$ is the $n \times n$ upper left corner of $H$.

Section 2 relates these $m$-functions to solutions of the second-order difference equation and obtains relations between $m_{ \pm}(z, n)$ and $m_{ \pm}(z, n+1)$ (of which (1.6) is a special case). Section 2 also contains some critical formulas expressing the diagonal Green's functions $G(z, n, n):=\left(\delta_{n},(H-z)^{-1} \delta_{n}\right)$ in terms of $m_{+}(z)$ and $m_{-}(z)$.

Section 4 contains one of the most intriguing results of this paper. In [48], Hochstadt proved the remarkable result that for a finite Jacobi matrix, a knowledge of all but the first $N c$ 's and the $N$-eigenvalues, that is, of $c_{N+1}, c_{N+2}, \ldots, c_{2 N-1}$ and $\lambda_{1}, \ldots, \lambda_{N}$, determines $H$ uniquely. In Section 4 , we extend this by showing that $c_{n+1}, \ldots, c_{2 N-1}$ and any $n$ eigenvalues of $H$ determine $H$ uniquely for any $n=1,2, \ldots, N$.

After a brief interlude in Section 5 obtaining the straightforward analog of Borg's twospectra theorem [11] (see also [12, 54, 55, 57, 59]) first considered in the Jacobi context by Hochstadt [46, 47] (see also [10, 27, 40, 41, 45, 48, 69]), we turn in Section 6 to the question of determining $H$ from a diagonal Green's function element $\left(\delta_{n},(H-z)^{-1} \delta_{n}\right)$ when $N<\infty$. If $n=1$ or $N$, the central inverse spectral theory result says $G(z, n, n)$ uniquely determines $H$. For other $n$, there are always at least $\left(\begin{array}{l}N-1 \\ n-1\end{array}\right)$ different $H$ 's compatible with a given $G(z, n, n)$. Generically, there are precisely that many $H$ 's. Section 6 has a complete analysis.

In a final Section 7 we present some results and conjectures about the inverse problem when $a_{n} \equiv 1$.

\section{§2. $m$-Function Formulas}

Let $H$ be a finite or semi-infinite Jacobi matrix of the type described in Section 1 . We begin by defining some special functions of a complex variable $z$ which we will call $\{P(z, n)\}_{n=1}^{N+1}$ and $\left\{\psi_{+}(z, n)\right\}_{n=0}^{N}$. The $P(z, n)$ 's are polynomials of degree $n-1$ defined by the pair of conditions

$$
\begin{gathered}
a_{n} P(z, n+1)+b_{n} P(z, n)+a_{n-1} P(z, n-1)=z P(z, n), \quad 1 \leq n<N+1, \\
P(z, 0)=0, \quad P(z, 1)=1 .
\end{gathered}
$$


(For convenience, we define $a_{N}:=1$ in order to define $P(z, N+1)$ in case $N<\infty$.) Clearly, (2.1) defines $P(z, n)$ inductively as a polynomial of the claimed degrees. Again, inductively it is clear that

$$
P(z, j+1)=\frac{1}{a_{1} \ldots a_{j}} z^{j}+\text { lower degree in } z .
$$

As explained in the appendix, the $P$ 's are intimately related to the spectral measure for $H$ (as defined in Section 1).

Proposition 2.1. ([7], p. 542)

$$
P(z, j+1)=\left(a_{1} \ldots a_{j}\right)^{-1} \operatorname{det}\left(z-H_{[1, j]}\right), \quad j \geq 1,
$$

where $H_{[1, j]}$ is the $j \times j$ matrix in the upper left corner of $H$.

Proof. By $(2.2), a_{1} \ldots a_{j} P(z, j+1)$ and $\operatorname{det}\left(z-H_{[1, j]}\right)$ are monic polynomials of degree $j$. Thus, it suffices to show they have the same zeros and multiplicities. But $P(z, j+1)=0$ if and only if there is a vector $v=\left(v_{1}, \ldots, v_{j}\right)$ with $v_{1}=1$ so that $\left(H_{[1, j]}-z\right) v=0$. As explained in Section 1, every eigenvector of $H_{[1, j]}$ has $v_{1} \neq 0$. Thus, the zeros of $P(z, j+1)$ are precisely the eigenvalues of $H_{[1, j]}$. Since the eigenvalues are simple, the multiplicities are all one.

In case $N<\infty, \psi_{+}(z, n)$ is defined via

$$
\begin{gathered}
a_{n} \psi_{+}(z, n+1)+b_{n} \psi_{+}(z, n)+a_{n-1} \psi_{+}(z, n-1)=z \psi_{+}(z, n), n=0, \ldots, N-1,(2 \\
\psi_{+}(z, N)=1, \quad \psi_{+}(z, N+1)=0,
\end{gathered}
$$

where again for convenience we define $a_{0}=1$ to enable us to define $\psi_{+}(z, 0)$. $\psi_{+}$is just like $P$ but run from the other end. By the same reasoning,

$$
\psi_{+}(z, N-j)=\frac{1}{a_{N-1} \ldots a_{N-j}} \operatorname{det}\left(z-H_{[N-j+1, N]}\right)
$$

is a polynomial of degree $j$.

In case $N=\infty, \psi_{+}(z, n)$ initially is only defined in the region $\operatorname{Im}(z) \neq 0$ by requiring (2.4) and

$$
\psi_{+}(z, 0)=1, \quad \sum_{n=0}^{\infty}\left|\psi_{+}(z, n)\right|^{2}<\infty .
$$

It is a standard argument that when $H$ is bounded and self-adjoint, there is a solution that is $\ell^{2}$ at infinity unique up to constant multiples (and everywhere non-vanishing so one can normalize it by $\left.\psi_{+}(z, 0)=1\right)$.

Given any two sequences $u(n), v(n)$, define the (modified) Wronskian $W(u, v)$ by

$$
W(u, v)(n)=a_{n}[u(n) v(n+1)-u(n+1) v(n)] .
$$

For any two solutions of (2.1), $W$ is constant. The Green's function is defined by $(1 \leq$ $m, n<N+1)$

$$
G(z, m, n)=\left(\delta_{m},(H-z)^{-1} \delta_{n}\right)
$$

for $\operatorname{Im}(z) \neq 0$. We will also sometimes use $(j \leq m, n \leq k)$

$$
G_{[j, k]}(z, m, n)=\left(\delta_{m},\left(H_{[j, k]}-z\right)^{-1} \delta_{n}\right) .
$$

We have the following standard formula: 


\section{Proposition 2.2.}

$$
G(z, m, n)=\left[W\left(P(z, \cdot), \psi_{+}(z, \cdot)\right)\right]^{-1} P(z, \min (m, n)) \psi_{+}(z, \max (m, n)) .
$$

Proof. One easily checks that if $G(z, m, n)$ is defined by $(2.8)$, then

$$
\sum_{k}\left(H_{m, k}-z \delta_{m, k}\right) G(z, k, n)=\delta_{m, n} .
$$

In the finite case, the choice of $P, \psi_{+}$ensures that the equation holds at the points where $n$ or $m$ equals 1 or $N$. In the infinite case, the choice of $P$ ensures the equation holds at $n$ or $m$ equals 1 , and the choice of $\psi_{+}$ensures that $\sum_{n} G(z, k, n) f_{n}$ is $\ell^{2}$ in $k$ for any finite support sequence $\left\{f_{n}\right\}$. In either case, it follows that $G(z, m, n)$ is indeed the matrix of the resolvent.

We can now define the most basic $m$-function (there will be more later),

$$
m(z)=\left(\delta_{1},(H-z)^{-1} \delta_{1}\right) .
$$

By (2.8), we claim

\section{Proposition 2.3.}

$$
m(z)=-\frac{\psi_{+}(z, 1)}{a_{0} \psi_{+}(z, 0)}
$$

Remark. By our convention, $a_{0}=1$, but we carry it along for the general definition of $m(z, n)$ later (cf. $(2.14))$.

Proof. $P(z, 0)=0, P(z, 1)=1$ so (2.8) becomes

$$
G(z, 1,1)=\frac{\psi_{+}(z, 1)}{-a_{0} \psi_{+}(z, 0)} .
$$

In terms of the spectral measure $d \rho$,

$$
m(z)=\int \frac{d \rho(\lambda)}{\lambda-z} .
$$

Theorem 2.4. If $N$ is finite, then

$$
m(z)=-\frac{\prod_{\ell=1}^{N-1}\left(z-\nu_{\ell}\right)}{\prod_{j=1}^{N}\left(z-\lambda_{j}\right)},
$$

where $\lambda_{1}<\cdots<\lambda_{N}$ are the eigenvalues of $H$ and $\nu_{1}<\cdots<\nu_{N-1}$ are the eigenvalues of $H_{[2, N]}$.

Proof. By (2.5) and (2.10),

$$
m(z)=-\frac{\operatorname{det}\left(z-H_{[2, N]}\right)}{\operatorname{det}(z-H)} .
$$

This can be viewed as a cofactor formula for the matrix elements of $(H-z)^{-1}$. 
Corollary 2.5. If $N$ is finite, $\left\{\lambda_{j}\right\}_{j=1}^{N} \cup\left\{\nu_{\ell}\right\}_{\ell=1}^{N-1}$ uniquely determine $H$. Any set of real $\lambda$ 's and $\nu$ 's are allowed as long as

$$
\lambda_{1}<\nu_{1}<\lambda_{2}<\nu_{2}<\cdots<\lambda_{N}
$$

Proof. By (2.12), the $\lambda$ 's and $\nu$ 's determine $m$, and then by (2.11), they determine $d \rho$. By Theorem A.6 (in the appendix), $d \rho$ determines the $a$ 's and $b$ 's. That any $\nu$ 's, $\lambda$ 's are allowed follows from the fact that if

$$
m(z)=\sum_{j=1}^{N} \frac{\alpha_{j}}{\lambda_{j}-z}
$$

then $\alpha_{j}>0$ for all $j$ is equivalent to (2.13).

Remark. The result (proven, e.g., in [10, 40, 41, 45-48]) is an analog of Borg's result for Sturm-Liouville operators that two spectra determine the potential. Its analog for $N=\infty$ (see [27]) is that Krein's spectral shift function for the pair $\left(H, H_{[2, \infty)}\right)$ determines $m(z)$ (cf. $[34,69])$.

Definition 2.6. $m_{+}(z, n)=\left(\delta_{n+1},\left(H_{[n+1, N]}-z\right)^{-1} \delta_{n+1}\right), n=0,1, \ldots, N-1$, where $H_{[n+1, N]}$ is interpreted as $H_{[n+1, \infty)}$ if $N=\infty$.

Thus, $m(z):=m_{+}(z, 0)$, and by the same calculation that led to $(2.10)$,

$$
m_{+}(z, n)=-\psi_{+}(z, n+1) /\left[a_{n} \psi_{+}(z, n)\right]
$$

¿From (2.4), we deduce the following Ricatti equation (more precisely, an analog of what is a Ricatti equation in the continuum case),

$$
a_{n}^{2} m_{+}(z, n)+\frac{1}{m_{+}(z, n-1)}=b_{n}-z
$$

It is also useful to have an analog of the $m$-function but starting at 1 instead of at $N$ or $\infty$.

Definition 2.7. $m_{-}(z, n)=\left(\delta_{n-1},\left(H_{[1, n-1]}-z\right)^{-1} \delta_{n-1}\right), n=2,3, \ldots, N+1$.

We immediately have analogs of (2.14) and (2.15), viz.,

$$
\begin{aligned}
& m_{-}(z, n)=-P(z, n-1) /\left[a_{n-1} P(z, n)\right] \\
& a_{n-1}^{2} m_{-}(z, n)+\frac{1}{m_{-}(z, n+1)}=b_{n}-z .
\end{aligned}
$$

The usefulness of having both $m_{+}(z)$ and $m_{-}(z)$ is that we can use them to express $G(z, n, n)$. We claim 
Theorem 2.8.

$$
\begin{aligned}
G(z, n, n) & =\frac{-1}{a_{n}^{2} m_{+}(z, n)+a_{n-1}^{2} m_{-}(z, n)+z-b_{n}} \\
& =\frac{-1}{a_{n-1}^{2} m_{-}(z, n)-\frac{1}{m_{+}(z, n-1)}} \\
& =\frac{-1}{a_{n}^{2} m_{+}(z, n)-\frac{1}{m_{-}(z, n+1)}}, \quad n=1,2, \ldots
\end{aligned}
$$

Proof. It suffices to prove (2.19), for then (2.18) follows from (2.15) and then (2.20) follows from (2.17).

To prove (2.19), use (2.8) evaluating the Wronskian at $n-1$ to see that

$$
\begin{aligned}
G(z, n, n) & =\frac{1}{a_{n-1}\left(\frac{P(z, n-1)}{P(z, n)}-\frac{\psi_{+}(z, n-1)}{\psi_{+}(z, n)}\right)} \\
& =\frac{1}{-a_{n-1}^{2} m_{-}(z, n)+\left(m_{+}(z, n-1)\right)^{-1}}
\end{aligned}
$$

by (2.14) and (2.16).

Theorem 2.9. Let $N \in \mathrm{N}$. At any eigenvalue $\lambda_{j}$ of $H$ we infer that

$$
m_{-}\left(\lambda_{j}, n+1\right)=\left[a_{n}^{2} m_{+}\left(\lambda_{j}, n\right)\right]^{-1}, \quad 1 \leq n \leq N-1,
$$

where equality in (2.21) includes the case that both sides equal infinity.

Proof. At first sight, this would seem to be a triviality. For $G(z, n, n)$ has poles at $\lambda_{j}$ and thus the denominator in (2.20) must vanish. But there is a subtlety. It can happen that at an eigenvalue $\lambda_{j}$ of $H, P\left(\lambda_{j}, n\right)=\psi_{+}\left(\lambda_{j}, n\right)=0$ and $G(z, n, n)$ then also vanishes at $\lambda_{j}$.

Thus we consider two cases: First $\varphi_{j}(n) \neq 0\left(\varphi_{j}\right.$ the eigenvector of $H$ associated with $\left.\lambda_{j}\right)$. In that case $G(z, n, n)$ has a pole as $z \rightarrow \lambda_{j}$ and so by $(2.20),(2.21)$ must hold (although both sides will be infinite if $\varphi_{j}(n+1)=0$ ).

In the second case, $\varphi_{j}(n)=0$. Then both sides of (2.21) are zero, and so (2.21) holds. (However, the denominator of $(2.20)$ is $\infty-\infty$ and happens to be $\infty$ so that $G(z, n, n)$ vanishes, but (2.21) still holds.)

\section{$\S 3$. Trace Formulas and a New Approach to the Inverse Problem}

In this section, we will use $m$-functions to show how to recover a Jacobi matrix from the spectral function $d \rho$. The more usual approach via orthogonal polynomials is sketched in the appendix. Our approach is new, although iterated $m$-functions are equivalent to a continued fraction expansion of $m(z)$, and so the work of Masson and Repka [61] is not unrelated to our approach.

We begin with 
Theorem 3.1. Near $z=\infty$,

$$
m(z)=-\frac{1}{z}-\frac{b_{1}}{z^{2}}-\frac{a_{1}^{2}+b_{1}^{2}}{z^{3}}+O\left(z^{-4}\right) .
$$

First Proof. By the basic definition of $m(z)$ (see (2.9)) and the norm convergent expansion (since $H$ is bounded):

$$
\begin{aligned}
(H-z)^{-1} & =-z^{-1}\left(1-z^{-1} H\right)^{-1} \\
& =-z^{-1}-z^{-2} H-z^{-3} H^{2}+O\left(z^{-4}\right) .
\end{aligned}
$$

We have

$$
m(z)=-z^{-1}-z^{-2}\left(\delta_{1}, H \delta_{1}\right)-z^{-3}\left\|H \delta_{1}\right\|^{2}+O\left(z^{-4}\right) .
$$

Clearly, $\left(\delta_{1}, H \delta_{1}\right)=b_{1}$ and $\left\|H \delta_{1}\right\|^{2}=\left\|a_{1} \delta_{2}+b_{1} \delta_{1}\right\|^{2}=a_{1}^{2}+b_{1}^{2}$.

Second Proof. By (2.15),

$$
m(z)=\frac{1}{b_{1}-z-a_{1}^{2} m_{+}(z, 1)} .
$$

But $m_{+}(z, 1)=-\frac{1}{z}+O\left(z^{-2}\right)$. Thus,

$$
\begin{aligned}
m(z) & =-\frac{1}{z}\left(1-\frac{b_{1}}{z}-\frac{a_{1}^{2}}{z^{2}}+O\left(z^{-3}\right)\right)^{-1} \\
& =-\frac{1}{z}\left(1+\frac{b_{1}}{z}+\frac{a_{1}^{2}}{z^{2}}+\left(\frac{b_{1}}{z}\right)^{2}+O\left(z^{-3}\right)\right) .
\end{aligned}
$$

In terms of the spectral measure $d \rho,(3.1)$ becomes

$$
\begin{aligned}
b_{1} & =\int \lambda d \rho(\lambda), \\
a_{1}^{2} & =\int \lambda^{2} d \rho(\lambda)-\left(\int \lambda d \rho(\lambda)\right)^{2},
\end{aligned}
$$

formulas implicit in the orthogonal polynomial approach.

In case $N<\infty$, there is a direct way to interpret (3.1) as generating trace formulas:

Theorem 3.2. Assume $N \in \mathrm{N}$ and let $\lambda_{1}, \ldots, \lambda_{N}$ be the eigenvalues of $H$ and $\nu_{1}, \ldots, \nu_{N-1}$ the eigenvalues of $H_{[2, N]}$. Then

$$
\begin{array}{r}
b_{1}=\sum_{j=1}^{N} \lambda_{j}-\sum_{\ell=1}^{N-1} \nu_{\ell}, \\
2 a_{1}^{2}+b_{1}^{2}=\sum_{j=1}^{N} \lambda_{j}^{2}-\sum_{\ell=1}^{N-1} \nu_{\ell}^{2} .
\end{array}
$$


Proof. Write (see (2.12))

$$
\begin{aligned}
m(z) & =-\frac{\prod_{\ell=1}^{N-1}\left(z-\nu_{\ell}\right)}{\prod_{j=1}^{N}\left(z-\lambda_{j}\right)}=-\frac{1}{z} \prod_{\ell=1}^{N-1}\left(1-\frac{\nu_{\ell}}{z}\right) \prod_{j=1}^{N}\left(1-\frac{\lambda_{j}}{z}\right)^{-1} \\
& =-\frac{1}{z}-\frac{\alpha}{z^{2}}-\frac{\beta}{z^{3}}+O\left(z^{-4}\right),
\end{aligned}
$$

where

$$
\begin{aligned}
& \alpha=\sum_{j=1}^{N} \lambda_{j}-\sum_{\ell=1}^{N-1} \nu_{\ell}, \\
& \beta=\sum_{j=1}^{N} \lambda_{j}^{2}+\sum_{j<k}^{N} \lambda_{j} \lambda_{k}+\sum_{\ell<m}^{N-1} \nu_{\ell} \nu_{m}-\sum_{j=1}^{N} \lambda_{j} \sum_{\ell=1}^{N-1} \nu_{\ell} .
\end{aligned}
$$

(3.6) is just (3.4), and using (3.6), (3.7) becomes

$$
\beta=\frac{1}{2} \sum_{j=1}^{N} \lambda_{j}^{2}-\frac{1}{2} \sum_{\ell=1}^{N-1} \nu_{\ell}^{2}+\frac{1}{2} \alpha^{2} .
$$

Thus,

$$
\sum_{j=1}^{N} \lambda_{j}^{2}-\sum_{\ell=1}^{N-1} \nu_{\ell}^{2}=2 \beta-\alpha^{2}=2 a_{1}^{2}+b_{1}^{2}
$$

by $(3.1)$.

Of course, (3.4), (3.5) have direct proofs in terms of traces since they just say that

$$
\begin{aligned}
\operatorname{Tr}(H)-\operatorname{Tr}\left(H_{[2, N]}\right) & =b_{1}, \\
\operatorname{Tr}\left(H^{2}\right)-\operatorname{Tr}\left(H_{[2, N]}^{2}\right) & =2 a_{1}^{2}+b_{1}^{2}
\end{aligned}
$$

and is one reason why (3.1) should be thought of as generating trace formulas. In the case of periodic Jacobi matrices, this strategy has been employed in [62].

There is another way to write (3.1) that doesn't require us to analyze $m(z)$ for large $z$. Define the $\xi$ function [33] by

$$
\xi(\lambda)=\frac{1}{\pi} \operatorname{Arg}(m(\lambda+i 0)) \quad \text { for a.e. } \lambda \in \mathrm{R} .
$$

Then if $\operatorname{supp}(d \rho)=\operatorname{spec}(H) \subset[\alpha, \beta]$, we infer that $\xi(\lambda)=0$ for $\lambda \leq \alpha$ and $\xi(\lambda)=1$ for $\lambda \geq \beta$. We claim 


\section{Theorem 3.3.}

$$
\begin{gathered}
b_{1}=\alpha+\int_{\alpha}^{\beta}(1-\xi(\lambda)) d \lambda \\
2 a_{1}^{2}+b_{1}^{2}=\alpha^{2}+\int_{\alpha}^{\beta} 2 \lambda(1-\xi(\lambda)) d \lambda .
\end{gathered}
$$

Remark. (3.11) is proven in [33] and the method used to prove it also proves (3.12). The proof below is not unrelated to that in [33].

Proof. By Theorem 3.1, the function $-z m(z)$ has the asymptotics near $\infty$

$$
-z m(z)=1+\frac{b_{1}}{z}+\frac{a_{1}^{2}+b_{1}^{2}}{z^{2}}+O\left(z^{-3}\right) .
$$

Using $\ln (1+x)=x-\frac{1}{2} x^{2}+O\left(x^{3}\right)$ for $|x|$ sufficiently small, we see that

$$
Q(z):=\ln (-z m(z))
$$

has the asymptotics,

$$
Q(z)=\frac{b_{1}}{z}+\frac{2 a_{1}^{2}+b_{1}^{2}}{2 z^{2}}+O\left(z^{-3}\right) \quad \text { as } z \rightarrow \infty .
$$

Notice that the right sides of (3.11), (3.12) are unchanged if $\beta$ is increased or $\alpha$ is decreased (since $\xi(\lambda)=1$ if $\lambda>\beta$ and $\xi(\lambda)=0$ if $\lambda<\alpha$ ), so we can assume that $0 \in(\alpha, \beta)$. Then $Q(z)$ is analytic in $C \backslash[\alpha, \beta]$ and on $(\alpha, \beta)$ :

$$
\begin{aligned}
\frac{1}{\pi} \operatorname{Im}(Q(\lambda+i 0)) & =\xi(\lambda), & & \lambda<0 \\
& =\xi(\lambda)-1, & & \lambda>0 .
\end{aligned}
$$

By (3.13), for $R$ sufficiently large,

$$
\begin{aligned}
b_{1} & =\frac{1}{2 \pi i} \oint_{|z|=R} Q(z) d z=-\int_{\alpha}^{\beta} \frac{1}{\pi} \operatorname{Im}(Q(\lambda+i 0)) d \lambda \\
& =-\int_{\alpha}^{0} d \lambda+\int_{\alpha}^{\beta}(1-\xi(\lambda)) d \lambda=(3.11)
\end{aligned}
$$

and

$$
\begin{aligned}
2 a_{1}^{2}+b_{1}^{2} & =\frac{1}{2 \pi i} \oint_{|z|=R} 2 z Q(z) d z=-\int_{\alpha}^{\beta} \frac{1}{\pi} 2 \lambda \operatorname{Im}(Q(\lambda+i 0)) d \lambda \\
& =-\int_{\alpha}^{0} 2 \lambda d \lambda+\int_{\alpha}^{\beta} 2 \lambda(1-\xi(\lambda)) d \lambda=(3.12) .
\end{aligned}
$$


(3.2)-(3.5), (3.8), (3.9), (3.11), (3.12), etc. clearly underscore that one can derive an infinite sequence of such trace formulas which are precisely the well-known invariants of the hierarchy of Toda lattices. A systematic approach to these trace formulas can be found, for instance, in $[13,19,31,69]$.

We can now describe the scheme for recovering $H$ from $d \rho$, or equivalently, from $m(z)=$ $\int d \rho(\lambda)(\lambda-z)^{-1}$ :

(i) Use the trace formulas (via (3.1) or (3.11), (3.12)) to recover $b_{1}$ and $a_{1}^{2}$.

(ii) Use (2.15), viz.,

$$
m_{+}(z, 1)=a_{1}^{-2}\left(b_{1}-z-\frac{1}{m(z)}\right)
$$

to find $m_{+}(z, 1)$ which is the $m$-function for $H_{[2, \infty)}$.

(iii) Use the trace formulas to find $b_{2}, a_{2}^{2}$ and then $(2.15)$ to find $m_{+}(z, 2), \ldots$, etc.

This clearly shows a given $d \rho$ can come from at most one $H$, since we have just described how to compute the $b_{j}$ and $a_{j}^{2}$ from $d \rho$. We want to prove existence via this method, that is, given any $d \rho$ of compact support, this method yields an $H$ which is bounded and whose spectral measure is precisely $d \rho$.

Lemma 3.4. Suppose that $m(z)=\int d \rho(\lambda)(\lambda-z)^{-1}$, where $d \rho$ is a probability measure on $[-C, C]$ whose support contains more than one point. Define

$$
b_{1}=\int \lambda d \rho(\lambda), \quad a_{1}^{2}=\int \lambda^{2} d \rho(\lambda)-b_{1}^{2}
$$

( $a_{1}^{2}$ is always strictly positive by the support hypothesis on $d \rho$ ). Define $m_{1}(z)$ by

$$
m_{1}(z)=a_{1}^{-2}\left[b_{1}-z-\frac{1}{m(z)}\right]
$$

Then

$$
m_{1}(z)=\int \frac{d \rho_{1}(\lambda)}{\lambda-z}
$$

where $d \rho_{1}$ is a probability measure also supported on $[-C, C]$. Moreover, $\rho$ is supported on exactly $N$ points if and only if $\rho_{1}$ is supported on exactly $(N-1)$ points.

Proof. By (3.14) and an expansion of a geometric series, (3.1) holds, so

$$
\widehat{m}(z):=(-m(z))^{-1}=z-b_{1}-\frac{a_{1}^{2}}{z}+O\left(z^{-2}\right) .
$$

Since $m(z)$ has $\operatorname{Im}(m(z))>0$ when $\operatorname{Im}(z)>0$ (we recall that $m$ is a Herglotz function), $\widehat{m}(z)=(-m(z))^{-1}$ has the same property. Moreover, $\widehat{m}(z)$ is analytic on $C \backslash[-C, C]$ since $m(\lambda)>0$ for $\lambda<-C$ and $m(\lambda)<0$ for $\lambda>C$. Thus, by the Herglotz representation theorem,

$$
\widehat{m}(z)=\hat{c}+\hat{d} z+\int \frac{d \hat{\rho}(\lambda)}{\lambda-z}
$$


for a measure $d \hat{\rho}$. By (3.16), $\hat{c}=-b_{1}, \hat{d}=1$, and $\int d \hat{\rho}(\lambda)=a_{1}^{2}$. Thus,

$$
m_{1}(z)=\int \frac{d \rho_{1}(\lambda)}{\lambda-z}
$$

and $d \rho_{1}=a_{1}^{-2} d \hat{\rho}$ is also a probability measure.

Since $d \rho$ is supported on $N$ points if and only if $m(z)$ is a ratio $P_{N-1}(z) / Q_{N}(z)$ of polynomials with $\operatorname{deg}\left(P_{N-1}(z)\right)=N-1, \operatorname{deg}\left(Q_{N}(z)\right)=N$, we obtain the last assertion.

Theorem 3.5. (三 Theorem A.6) Every $N$-point probability measure arises as the spectral measure of a unique $N \times N$ Jacobi matrix. Every probability measure of bounded and infinite support arises as the spectral measure of a unique semi-infinite bounded Jacobi matrix.

Proof. By iterating the $\rho \rightarrow \rho_{1}$ procedure of the lemma, we can find suitable $a_{j}^{2}, b_{j}$ inductively. If $d \rho$ has $N$-point support, the process terminates after $N-1$ steps where $d \rho_{N}$ has a single point, and we define $b_{N}$ to be that point. If $d \rho$ has infinite support, the process continues indefinitely. Because $\operatorname{supp}(d \rho) \subseteq[-C, C]$, we infer $\operatorname{supp}\left(d \rho_{1}\right) \subseteq[-C, C],\left|a_{1}\right|$ and $\left|b_{1}\right|$ are bounded by $C$, and so $H$ is bounded.

Let $d \tilde{\rho}$ be the spectral measure for the $H$ that has just been constructed. We will show $d \rho=d \tilde{\rho}$, thereby completing the proof.

Let $\tilde{m}(z)=\int d \tilde{\rho}(\lambda)(\lambda-z)^{-1}$. Then by construction,

$$
\tilde{m}(z)=\frac{-1}{z-b_{1}+a_{1}^{2}\left[\frac{-1}{z-b_{2}+a_{2}^{2} \cdots}\right]} .
$$

That is, $m$ and $\tilde{m}$ have identical partial fraction expansions although a priori the remainders could be different. This means that the Taylor series for $\tilde{m}(z)$ near $z=\infty$ agrees with that for $m$ near $z=\infty$ so $m(z)=\tilde{m}(z)$, and hence $d \rho=d \tilde{\rho}$.

Remark. The Taylor series for $m(z)$ only converges in the region $|z|>C$, where $C=$ $\max (\operatorname{supp}(d \rho))$. But $H_{[1, N]} \rightarrow H$ strongly, so $\left(\delta_{1},\left(H_{[1, N]}-z\right)^{-1} \delta_{1}\right):=m_{N}(z) \rightarrow m(z)$ as $N \rightarrow \infty$ for all $z \notin \operatorname{supp}(d \rho)$, where $m_{N}(z)=P_{N-1}(z) / Q_{N}(z)$ is a ratio of polynomials of degree $N-1$ and $N$. By the above argument, the Taylor series agree near infinity to order $2 N$, that is, $m_{N}$ is the $[N-1, N]$ Padé approximant, which we see converges everywhere outside of $\operatorname{supp}(d \rho)$. See Baker and Graves-Morris [5] for further discussion of the Padé approximants.

The continuum analog of the orthogonal polynomial approach of the appendix is the Gel'fand-Levitan [29] inverse spectral theory which is a kind of continuum orthonormalization. It would be very interesting to find a continuum analog of the $m$-function approach to inverse problems that we discussed in this section.

As an application of the $m$-function approach to inverse problems, we prove the following (which can also be obtained via orthogonal polynomials): 
Theorem 3.6. Fix $N \in \mathrm{N}$. Consider the following parametrizations of $N \times N$ Jacobi matrices:

$$
\begin{aligned}
& \text { (i) }\left\{a_{n}\right\}_{n=1}^{N-1} \cup\left\{b_{n}\right\}_{n=1}^{N}, \quad\left(a_{n}>0\right) \text {. } \\
& \text { (ii) }\left\{\lambda_{j}\right\}_{j=1}^{N} \cup\left\{\nu_{\ell}\right\}_{\ell=1}^{N-1}, \quad\left(\lambda_{1}<\nu_{1}<\lambda_{2}<\cdots<\nu_{N-1}<\lambda_{N}\right) \text {. } \\
& \text { (iii) }\left\{\lambda_{j}\right\}_{j=1}^{N} \cup\left\{\alpha_{j}\right\}_{j=1}^{N}, \quad\left(\lambda_{1}<\cdots<\lambda_{N}, \alpha_{j}>0, \sum_{k=1}^{N} \alpha_{k}=1\right) \text {. }
\end{aligned}
$$

Here $\lambda_{j}$ are the eigenvalues of $H, \nu_{\ell}$ are the eigenvalues of $H_{[2, n]}$, and the $\alpha$ 's are the residues of the poles in $m$ so $m(z)=\sum_{j=1}^{N} \alpha_{j}\left(\lambda_{j}-z\right)^{-1}\left(\right.$ or $\left.d \rho(\lambda)=\sum \alpha_{j} \delta\left(\lambda-\lambda_{j}\right) d \lambda\right)$. The maps between these parameters are real bianalytic diffeomorphisms.

Remark. There are $2 N-1$ parameters. These appear to be $2 N$ in (iii) but the fact that $\sum_{j=1}^{N} \alpha_{j}=1$ eliminates one parameter.

Proof. It is well known and elementary (the determinant of the Jacobian matrix is just $\left.\pm \prod_{j<k}\left(\lambda_{j}-\lambda_{k}\right)^{-1}\right)$ that the map from the $N$ coefficients of a monic polynomial $P_{N}(\lambda)$ of degree $N$ to the roots $\lambda_{1}, \ldots, \lambda_{N}$ of that polynomial is a bianalytic diffeomorphism in the region where the roots are all real and distinct. This immediately implies that the map from (i) to (ii) is real analytic. The map from (ii) to (iii) is rational since $\alpha_{j}=\prod_{\ell=1}^{N-1}\left(\lambda_{j}-\nu_{\ell}\right) \prod_{k \neq j}^{N}\left(\lambda_{j}-\lambda_{k}\right)^{-1}$. That means we need only show that the map from (iii) to (i) is real analytic.

Since $b_{1}=\sum_{j=1}^{N} \alpha_{j} \lambda_{j}$ and $a_{1}^{2}=\left(\sum_{j=1}^{N} \alpha_{j} \lambda_{j}^{2}\right)-b_{1}^{2}$, those are analytic functions. Moreover, the $\nu_{\ell}$ are the roots of the polynomial $\sum_{j=1}^{N} \alpha_{j} \prod_{k \neq j}\left(z-\lambda_{k}\right)$ and so real analytic in $\left(\lambda_{j}, \alpha_{j}\right)$ by the first sentence in this proof. $m_{+}(z, 1)$ has the form $\sum_{\ell=1}^{N-1} \beta_{\ell}\left(\nu_{\ell}-z\right)^{-1}$, where $\beta_{\ell}=\left[a_{1}^{2} m^{\prime}\left(\nu_{\ell}\right)\right]^{-1}$ is clearly analytic in the $\lambda^{\prime}$ 's and $\alpha$ 's. Thus following the $m$-function reconstruction shows that the $a$ 's and $b$ 's are real analytic functions of the $\lambda$ 's and $\alpha$ 's.

\section{$\S 4$. Recovery of a Matrix From Parts of the Matrix and Additional Spectral Information}

In [48], Hochstadt proved the following remarkable theorem (see (1.3)) for the definition of $c_{j}$ ):

Theorem 4.1. (三 Theorem A.7) Let $N \in \mathrm{N}$. Suppose that $c_{N+1}, \ldots, c_{2 N-1}$ are known, as well as the eigenvalues $\lambda_{1}, \ldots, \lambda_{N}$ of $H$. Then $c_{1}, \ldots, c_{N}$ are uniquely determined.

Hochstadt's proof is sketched in the appendix (but in "reflected" coordinates, i.e., $c_{1}, \ldots, c_{N-1}$ are assumed to be known). Our goal in this section is to prove

Theorem 4.2. Suppose that $1 \leq j \leq N$ and $c_{j+1}, \ldots, c_{2 N-1}$ are known, as well as $j$ of the eigenvalues. Then $c_{1}, \ldots, c_{j}$ are uniquely determined.

Remarks. 1. One need not know which of the $j$ eigenvalues one has. 
2. Hochstadt-Lieberman [49] proved an analog of Hochstadt's theorem in the continuum case. In [36], we will prove continuum analogs of Theorem 4.2; [35] contains an application of our $m$-function technique to uniqueness theorems for Schrödinger operators $H$ on $\mathrm{R}$ in terms of the reflection coefficient for right incidence under the assumption that $H$, restricted to the half-line $(0, \infty)$, has an absolutely continuous (a.c.) component in its spectrum.

3. In some sense, Hochstadt's proof [48] goes from the edge inward and ours goes from the inside toward the edge.

We will need some elementary facts about rational functions. Let $f=P / Q$ be a ratio of polynomials. We define the degree $\operatorname{deg}(f)$ of $f$ to be $\operatorname{deg}(P)+\operatorname{deg}(Q)$. If $P$ and $Q$ are both monic, we will say that $f$ is monic.

Lemma 4.3. Suppose $f_{1}=P_{1} / Q_{1}, f_{2}=P_{2} / Q_{2}$, where $\operatorname{deg}\left(P_{1}\right)=\operatorname{deg}\left(P_{2}\right)$ and $\operatorname{deg}\left(Q_{1}\right)=$ $\operatorname{deg}\left(Q_{2}\right)$, and $d=\operatorname{deg}\left(f_{i}\right)$.

(i) If $f_{1}$ and $f_{2}$ agree at $d+1$ points in $\mathrm{C}$, then $f_{1}=f_{2}$.

(ii) If $f_{1}$ and $f_{2}$ are both monic and they agree at $d$ points in $C$, then $f_{1}=f_{2}$.

Remark. "agree" here allows the possibility that both are infinite at the same point.

Proof. If $f_{1}(z)=f_{2}(z)$, then $P_{1}(z) Q_{2}(z)-P_{2}(z) Q_{1}(z)=0$ (even if both values are infinite, since then $Q_{1}=Q_{2}=0$ ). In case (i), $P_{1} Q_{2}-Q_{1} P_{2}$ has degree $d$. In case (ii), the leading terms cancel and $P_{1} Q_{2}-Q_{1} P_{2}$ has degree $d-1$. The lemma follows from the fact that if a polymonial $R_{d_{0}}$ of degree $d_{0}$ vanishes at $d_{0}+1$ points, then $R_{d_{0}} \equiv 0$.

Proof of Theorem 4.2. Suppose first that $j$ is odd so $j=2 n-1$, and $b_{1}, \ldots, a_{n-1}, b_{n}$ are unknown, but $a_{n}, b_{n+1}, \ldots, b_{N}$ are known, as well as $j$ eigenvalues which we will denote $\lambda_{1}, \ldots, \lambda_{2 n-1}$. By $(2.21)$

$$
-m_{-}\left(\lambda_{j}, n+1\right)=\left[-a_{n}^{2} m_{+}\left(\lambda_{j}, n\right)\right]^{-1} .
$$

By definition, $m_{+}(z, n)$ is determined by $H_{[n+1, N]}$ and so by $b_{n+1}, a_{n+1}, \ldots, b_{N}$. Thus, $\left[-a_{n}^{2} m_{+}\left(\lambda_{j}, n\right)\right]^{-1}$ are known numbers.

By the analog of Theorem 2.4 (see also $(2.16)),-m^{-}(n+1, z)$ is a ratio $P_{n-1}(z) / Q_{n}(z)$ of polynomials, where $\operatorname{deg}\left(P_{n-1}(z)\right)=n-1$ and $\operatorname{deg}\left(Q_{n}(z)\right)=n$, and each is monic. By part (ii) of Lemma 4.3, the values of such a monic rational function of degree $2 n-1$ is determined by its values at the $2 n-1$ points $\lambda_{1}, \ldots, \lambda_{2 n-1}$. Once we know $m_{-}(z, n+1)$, $b_{1}, a_{1}, \ldots, b_{n}$ are determined by Corollary 2.5 .

Suppose next that $j$ is even so $j=2 n$, and $a_{n}$ moves from the known group to the unknown group. We can use

$$
-a_{n}^{2} m_{-}\left(\lambda_{j}, n+1\right)=\left[-m_{+}\left(\lambda_{j}, n\right)\right]^{-1}
$$

to conclude that we know $f(z):=-a_{n}^{2} m_{-}(z, n+1)$ at the $2 n$ points $\lambda_{1}, \ldots, \lambda_{2 n} . f(z)$ is no longer monic, but it is of degree $2 n-1$ and so its values at $2 n$ points determine it uniquely by part (i) of Lemma 4.3 . Once we know $-a_{n}^{2} m_{-}(z, n+1)$, we can obtain $a_{n}^{2}$ by $\lim _{|z| \rightarrow \infty}\left[-z m_{-}(z, n+1)\right]=1$ and then $b_{1}, a_{1}, \ldots, b_{n}$ by Corollary 2.5 . 
Example 4.4. $(j=1)$ We use $m_{-}(z, 2)=\left(\delta_{1},\left(H_{[1,1]}-z\right)^{-1} \delta_{1}\right)=\left(b_{1}-z\right)^{-1}$. Then

$$
b_{1}=\lambda_{1}+a_{1}^{2} m_{+}\left(\lambda_{1}, 1\right)
$$

This has a solution as long as $m_{+}\left(\lambda_{1}, 1\right) \neq \infty$. The only forbidden values for $\lambda_{1}$ are the obvious ones, namely, the eigenvalues $\nu_{\ell}$ of $H_{[2, N]}$ which we know must be unequal to the $\lambda_{j}$ 's.

Example 4.5. $(j=2)$ We get

$$
b_{1}=\lambda_{j}+a_{1}^{2} m_{+}\left(\lambda_{j}, 1\right), \quad j=1,2
$$

$m_{+}\left(\lambda_{j}, 1\right) \neq \infty$ is still required, but we also need that

$$
-\frac{m_{+}\left(\lambda_{2}, 1\right)-m_{+}\left(\lambda_{1}, 1\right)}{\lambda_{2}-\lambda_{1}}
$$

which equals $a_{1}^{-2}$, must be positive. This avoids two eigenvalues between a single pair of eigenvalues of $H_{[2, N]}$ but requires a lot more. There are severe restrictions in the $\lambda_{j}$ 's for existence (see, e.g., the discussion in [23]). As $j$ increases, these become more complicated.

\section{$\S 5$. Reconstruction of a Finite Jacobi Matrix From Two Spectra}

Borg [11] proved a famous theorem that the spectra for two boundary conditions of a bounded interval regular Schrödinger operator uniquely determine the potential. Later refinements (see, e.g., $[12,54,55,57,59]$ ) imply that they even determine the two boundary conditions.

In this section, we consider analogs of this result for a finite Jacobi matrix. Such analogs were first considered by Hochstadt [46, 47] (see also [10, 27, 40, 41, 45, 48]). In one sense, the fact that the eigenvalues of $H_{[1, N]}$ and $H_{[2, N]}$ determine $H$ is such a two-spectrum result and, indeed, it can be viewed as Theorem 5.2 below for $b=\infty$. Our results in this section are straightforward adaptations of known results for the continuum or the semi-infinite case, but the ability to determine parameters by counting sheds light on facts like the one that the lowest eigenvalue in the Borg result is not needed under certain circumstances.

Given $H$, an $N \times N$ Jacobi matrix, define $H(b)$ to be the Jacobi matrix where all $a$ 's and $b$ 's are the same as $H$, except $b_{1}$ is replaced by $b_{1}+b$, that is,

$$
H(b)=H+b\left(\delta_{1}, \cdot\right) \delta_{1}
$$

Theorem 5.1. The eigenvalues $\lambda_{1}, \ldots, \lambda_{N}$ of $H$, together with $b$ and $N-1$ eigenvalues $\lambda(b)_{1}, \ldots, \lambda(b)_{N-1}$ of $H(b)$, determine $H$ uniquely.

Remark. Again it is irrelevant which $N-1$ eigenvalues of the $N$ eigenvalues of $H(b)$ are known. 
Proof. Choosing $a_{0}=1$, we have

$$
m(z)=-\psi_{+}(z, 1) / \psi_{+}(z, 0)
$$

and

$$
\psi_{+}(z, 0)+\left(b_{1}-z\right) \psi_{+}(z, 1)+a_{1} \psi_{+}(z, 2)=0 .
$$

It follows that $z$ is an eigenvalue of $H(b)$ if and only if

$$
\psi_{+}(z, 0)=b \psi_{+}(z, 1)
$$

that is, if and only if

$$
m(z)=-\frac{1}{b}
$$

(a standard result in the general theory of rank-one perturbations [65]).

Write $m(z)=-P_{N-1}(z) / Q_{N}(z)$, where $P_{N-1}(z)$ and $Q_{N}(z)$ are monic polynomials of degree $N-1$ and $N$, respectively. $Q_{N}(z)=\prod_{j=1}^{N}\left(z-\lambda_{j}\right)$ is known and

$$
P_{N-1}\left(\lambda(b)_{k}\right)=b^{-1} \prod_{j=1}^{N}\left(\lambda(b)_{k}-\lambda_{j}\right), \quad 1 \leq k \leq N-1
$$

are also known. Since the values of a monic polynomial $P_{d}(z)$ of degree $d$ at $d$ points uniquely determine $P_{d}(z)$ by Lagrange interpolation, $\lambda(b)_{1}, \ldots, \lambda(b)_{N-1}$ uniquely determine $P_{N-1}(z)$. The solution of the inverse problem, given $-P_{N-1}(z) / Q_{N}(z)$, and hence $m(z)$, then determines $H$ uniquely.

Theorem 5.2. The eigenvalues $\lambda_{1}, \ldots, \lambda_{N}$ of $H$, together with the $N$ eigenvalues $\lambda(b)_{1}, \ldots, \lambda(b)_{N}$ of some $H(b)$ (with $b$ unknown), determine $H$ and $b$.

Proof. Following the proof of Theorem 5.1, we have a monic polynomial $P_{N-1}(z)$, an unknown $\beta:=\frac{1}{b}$, and

$$
P_{N-1}\left(\lambda(b)_{k}\right)=\beta \prod_{j=1}^{N}\left(\lambda(b)_{k}-\lambda_{j}\right)
$$

Let

$$
R_{N}(z)=\beta \prod_{j=1}^{N}\left(z-\lambda_{j}\right)-P_{N-1}(z) .
$$

Since $R_{N}(z)=\beta z^{N}+$ lower-order terms and $R_{N}\left(\lambda(b)_{k}\right)=0,1 \leq k \leq N$, we have

$$
R_{N}(z)=\beta \prod_{j=1}^{N}\left(z-\lambda(b)_{j}\right)
$$

Since $P_{N-1}(z)$ is monic of degree $N-1$,

$$
R_{N}(z)=\beta z^{N}-\left(\beta \sum_{j=1}^{N} \lambda_{j}+1\right) z^{N-1}+\cdots
$$


on the one hand and

$$
R_{N}(z)=\beta z^{N}-\left(\beta \sum_{j=1}^{N} \lambda(b)_{j}\right) z^{N-1}+\cdots
$$

on the other. It follows that

$$
\beta=\frac{1}{\sum_{j=1}^{N}\left(\lambda(b)_{j}-\lambda_{j}\right)}=b^{-1} .
$$

Once $\beta$ is known, $R_{N}(z)$ determines $P_{N-1}(z)$, and thus $m(z)$ and $H$. (5.3) then determines $b$.

Remark. Since

$$
b=\operatorname{Tr}(H(b)-H)=\sum_{j=1}^{N}\left(\lambda(b)_{j}-\lambda_{j}\right),
$$

we can a priori deduce $b$ from the $\lambda(b)$ 's and $\lambda$ 's and so deduce Theorem 5.2 from Theorem 5.1. Of course, (5.3) is just an expression of this trace formula. Notice that the parameter counting works out. In Theorem $5.1,2 n-1$ eigenvalues determine $2 n-1$ parameters; and in Theorem 5.2, $2 n$ eigenvalues determine $2 n$ parameters.

\section{$\S 6$. The Interior Inverse Problem $(N<\infty)$}

The basic inverse spectral theorems (Theorem 3.5 resp. A.6) show that $\left(\delta_{1},(H-z)^{-1} \delta_{1}\right)$ determines $H$ uniquely. In this section, we take $N \in \mathrm{N}, 1 \leq n \leq N$, and ask whether $\left(\delta_{n},(H-z)^{-1} \delta_{n}\right)$ determines $H$ uniquely. For notational convenience, we occasionally allude to $G(z, n, n)$ as the $n n$ Green's function in the remainder of this section. The $n=1$ result can be summarized via:

Theorem 6.1. $\left(\delta_{1},(H-z)^{-1} \delta_{1}\right)$ has the form $\sum_{j=1}^{N} \alpha_{j}\left(\lambda_{j}-z\right)^{-1}$ with $\lambda_{1}<\cdots<$ $\lambda_{N}, \sum_{j=1}^{N} \alpha_{j}=1$ and each $\alpha_{j}>0$. Every such sum arises as the 11 Green's function of an $H$ and of exactly one such $H$.

For general $n$, define $\tilde{n}=\min (n, N+1-n)$. Then we will prove the following theorems: Theorem 6.2. $\left(\delta_{n},(H-z)^{-1} \delta_{n}\right)$ has the form $\sum_{j=1}^{k} \alpha_{j}\left(\lambda_{j}-z\right)^{-1}$ with $k$ one of $N, N-$ $1, \ldots, N-\tilde{n}+1$ and $\lambda_{1}<\cdots<\lambda_{k}, \sum_{j=1}^{k} \alpha_{j}=1$ and each $\alpha_{j}>0$. Every such sum arises as the $n n$ Green's function of at least one $H$.

Theorem 6.3. If $k=N$, then precisely $\left(\begin{array}{l}N-1 \\ n-1\end{array}\right) H$ 's yield the given $n n$ Green's function.

Theorem 6.4. If $k<N$, then infinitely many $H$ 's yield the given $n n$ Green's function. Indeed, the inverse spectral family is a collection of $\left(\begin{array}{c}k-1 \\ N-k\end{array}\right)\left(\begin{array}{c}k-1-N+k \\ n-1-N+k\end{array}\right)$ disjoint manifolds, each of dimension $N-k$ and diffeomorphic to an $(N-k)$-dimensional open ball.

Proofs. Consider first the case $k=N$ (which is generic; $k<N$ occurs in a set of Jacobi matrices of codimension 1). Let $\mu_{1}<\cdots<\mu_{N-1}$ be the zeros of $G(z, n, n):=$ 
$\sum_{j=1}^{N} \alpha_{j}\left(\lambda_{j}-z\right)^{-1}$. Then

$$
-G(z, n, n)^{-1}=z-b+\sum_{\ell=1}^{N-1} \frac{\beta_{\ell}}{\mu_{\ell}-z},
$$

where $b, \mu_{\ell} \in \mathrm{R}$, and $\beta_{\ell}>0$ are determined by the $\alpha$ 's and $\lambda$ 's. By (2.18),

$$
-G(z ; n, n)^{-1}=z-b_{n}+a_{n}^{2} m_{+}(z, n)+a_{n-1}^{2} m_{-}(z, n) .
$$

$m_{-}(z, n)=\left(\delta_{n-1},\left(H_{[1, n-1]}-z\right)^{-1} \delta_{n-1}\right)$ determines $H_{[1, n-1]}$ uniquely (by Theorem 3.5) and has the form

$$
m_{-}(z, n)=\sum_{j=1}^{n-1} \frac{\gamma_{j}}{e_{j}-z}, \quad \gamma_{j}>0
$$

where $\sum_{j=1}^{n-1} \gamma_{j}=1$ and the $e_{j}$ 's are the eigenvalues of $H_{[1, n-1]}$. Similarly, $m_{+}(z, n)$ $=\left(\delta_{n+1},\left(H_{[n+1, N]}-z\right)^{-1} \delta_{n+1}\right)$ determines $H_{[n+1, N]}$ uniquely and has the form

$$
m_{+}(z, n)=\sum_{j=1}^{N-n} \frac{\kappa_{j}}{f_{j}-z}, \quad \kappa_{j}>0
$$

where $\sum_{j=1}^{N-n} \kappa_{j}=1$ and the $f_{j}$ 's are the eigenvalues of $H_{[n+1, N]}$. Comparing (6.1)-(6.4), we see that $\left\{\mu_{\ell}\right\}_{\ell=1}^{N-1}=\left\{e_{j}\right\}_{j=1}^{n-1} \cup\left\{f_{j}\right\}_{j=1}^{N-n}$. We can choose which $\mu_{\ell}$ are to be $e_{j}$ in $\left(\begin{array}{l}N-1 \\ n-1\end{array}\right)$ ways. Once we make the choice,

$$
a_{n-1}^{2}=\sum_{\ell \text { so } \mu_{\ell} \text { is an } e_{j}} \beta_{\ell} \quad \text { and } \quad a_{n}^{2}=\sum_{\ell \text { so } \mu_{\ell} \text { is an } f_{j}} \beta_{\ell}
$$

and $m_{ \pm}(z, n)$ are determined. But $H_{[1, n-1]}, H_{[n+1, N]}$, and $a_{n-1}, b_{n}, a_{n}$ determine $H$. Thus for each choice, we can uniquely determine $H$. Moreover, since any sums of the form (6.3), (6.4) are legal for $m_{ \pm}(z, n)$, we have existence for each of the $\left(\begin{array}{l}N-1 \\ n-1\end{array}\right)$ choices.

$k=N$ if and only if all the eigenfunctions $\varphi_{j}(n)$ are non-vanishing at $n$. Eigenfunctions obey the boundary conditions at both ends, so if $\varphi_{j}(n)$ vanishes, so do $P(z, n)$ and $\psi_{+}(z, n)$, which are polynomials of degree $n-1$ and $N-n$; so at most $\min (n-1, N-n):=\tilde{n}-1$ eigenvalues of $H$ can fail to contribute to $G(z, n, n)$, that is, at least $N-\tilde{n}+1$ eigenvalues must contribute, that is, $k$ is one of $N, N-1, \ldots, N-\tilde{n}+1$. Eigenvalues that don't contribute are zeros of $G(z, n, n)$ and simultaneously eigenvalues of $H_{[1, n-1]}$ and $H_{[n+1, N]}$.

Thus if $k<N$, the $k-1$ poles of $-G(z, n, n)^{-1}$ are in three sets. $n_{0}:=N-k$ are eigenvalues of both $H_{[1, n-1]}$, and $H_{[n+1, N]}, n_{1}:=n-1-(N-k)$ are eigenvalues of $H_{[1, n-1]}$ alone, and $n_{2}:=(N-n)-(N-k)=k-n$ are eigenvalues of $H_{[n+1, N]}$ alone. Notice that $N>k \geq N-\tilde{n}+1$ implies $n_{0}>0, n_{1} \geq 0, n_{2} \geq 0$ and that $n_{0}+n_{1}+n_{2}=k-1$, $n_{0}+n_{1}=n-1$, and $n_{0}+n_{2}=N-n$. To reconstruct $m_{ \pm}(z, n)$ given $-G(z, n, n)^{-1}$, we have to make two sets of choices:

(i) Figure out which of $\mu_{1}, \ldots, \mu_{k-1}$ lie in each of the three sets. This yields $\left(\begin{array}{c}k-1 \\ n_{0}\end{array}\right)\left(\begin{array}{c}k-1-n_{0} \\ n_{1}\end{array}\right)=$ $\frac{(k-1) !}{n_{0} ! n_{1} ! n_{2} !}$ discrete choices. 
(ii) For each of the $n_{0} \mu_{\ell}$ 's in the set of common eigenvalues, we must pick a decomposition

and then take

$$
\beta_{\ell}=\beta_{\ell}^{(1)}+\beta_{\ell}^{(2)}, \beta_{\ell}^{(j)}>0
$$

$$
a_{n}^{2} m_{+}(z, n)=\sum_{\substack{\ell \text { so that } \\
\mu_{\ell} \text { is solely an } \\
H_{[n+1, N]} \text { eigenvalue }}} \frac{\beta_{\ell}}{\mu_{\ell}-z}+\sum_{\begin{array}{c}
\ell \text { so that } \\
\mu_{\ell} \text { is a } \\
\text { common eigenvalue }
\end{array}} \frac{\beta_{\ell}^{(1)}}{\mu_{\ell}-z}
$$

and

$$
a_{n-1}^{2} m_{-}(z, n)=\sum_{\substack{\ell \text { so that } \\
\mu_{\ell} \text { is solely an } \\
H_{[1, n-1]} \text { eigenvalue }}} \frac{\beta_{\ell}}{\mu_{\ell}-z}+\sum_{\begin{array}{c}
\ell \text { so that } \\
\mu_{\ell} \text { is a } \\
\text { common eigenvalue }
\end{array}} \frac{\beta_{\ell}^{(2)}}{\mu_{\ell}-z} .
$$

Every such choice yields an acceptable $H$. Since the map from poles and residues to matrices is a diffeomorphism (Theorem 3.6), the $\frac{(k-1) !}{n_{0} ! n_{1} ! n_{2} !}$ disjoint sets of poles and $\times,\left(0, \beta_{\ell}\right)$ residues lead to that number of manifolds diffeomorphic to the $n_{0}$-dimensional open ball.

\section{$\S 7$. The Discrete Schrödinger Inverse Spectral Problem}

A Jacobi matrix with all $a_{n}=1$ is called a discrete Schrödinger operator. The inverse problem for such operators is open, that is, there are no effective conditions on a spectral measure $d \rho$ that tell us that its associated Jacobi matrix has all $a_{n}=1$. (The isospectral manifold of general Jacobi matrices with $a_{n} \in \mathrm{R}$ is discussed in [70].)

Consider the finite case, $N \in \mathrm{N}$. The number $N$ of free parameters $\left\{b_{n}\right\}_{n=1}^{N}$ equals exactly the number of eigenvalues $\left\{\lambda_{j}\right\}_{j=1}^{N}$. The natural inverse problem is from $\lambda$ 's to $b$ 's. We do not have a complete solution, but have a number of conjectures and comments which we make in this section. $\lambda_{1}<\lambda_{2}<\cdots<\lambda_{N}$ are the eigenvalues of $H$. For any $\mathbf{b}=\left(b_{1}, \ldots b_{N}\right) \in \mathrm{R}^{N}$, define $\Lambda(\mathbf{b})=\left(\lambda_{1}, \ldots, \lambda_{N}\right) \in \mathrm{R}^{N}$ as the eigenvalues. Let $S_{N}=\operatorname{Ran}(\Lambda)$.

Main Conjecture 7.1. $S_{N}$ is a closed set in $\mathrm{R}^{N}$ whose interior $S_{N}^{\text {int }}$ is dense in $S_{N}$. For any $\boldsymbol{\lambda} \in S_{N}^{\text {int }}, \Lambda^{-1}(\boldsymbol{\lambda})$ contains $N$ ! points. For any $\boldsymbol{\lambda} \in \partial S_{N}, \Lambda^{-1}(\boldsymbol{\lambda})$ contains fewer than $N$ ! points.

Thus, we believe that $\Lambda^{-1}\left[S_{N}^{\text {int }}\right]$ is an $N$ !-fold cover of $S_{N}^{\text {int }}$, but it is likely an uninteresting one.

Conjecture 7.2. $\Lambda^{-1}\left[S_{N}^{\text {int }}\right]$ is a union of $N$ ! disjoint sets. On each of them, $\Lambda$ is a diffeomorphism to $S_{N}^{\text {int }}$.

In the complex domain, things are more interesting. There is a small neighborhood, $D$, of $\mathrm{R}^{N}$ in $\mathrm{C}^{N}$ to which $\Lambda$ can be analytically continued and on which $\lambda_{j} \neq \lambda_{k}$ still holds. Introduce

$$
\tilde{S}_{N}=\Lambda[D] \quad \text { and } \quad B=\left\{\boldsymbol{\lambda} \in \tilde{S}_{N} \mid \Lambda^{-1}[\boldsymbol{\lambda}] \text { has ordinality less than } N !\right\} .
$$


Conjecture 7.3. $B$ has real codimension $2 . \Lambda^{-1}\left[\tilde{S}_{N} \backslash B\right]$ is connected and is an $N$ !-cover of $\tilde{S}_{N} \backslash B$.

Thus, $\Lambda^{-1}$ is a ramified cover of $\tilde{S}_{N}$. We begin with an analysis of the case $N=2$, so $H=\left(\begin{array}{cc}b_{1} & 1 \\ 1 & b_{2}\end{array}\right)$. Then

$$
\Lambda(\mathbf{b})=\left(\frac{b_{1}+b_{2}}{2}-\sqrt{\left(\frac{b_{1}-b_{2}}{2}\right)^{2}+1}, \frac{b_{1}+b_{2}}{2}+\sqrt{\left(\frac{b_{1}-b_{2}}{2}\right)^{2}+1}\right) .
$$

Then $S_{2}=\left\{\left(\lambda_{1}, \lambda_{2}\right) \in \mathrm{R}^{2} \mid \lambda_{2} \geq \lambda_{1}+2\right\} . \partial S_{2}=\left\{\left(\lambda_{1}, \lambda_{2}\right) \in \mathrm{R}^{2} \mid \lambda_{2}=\lambda_{1}+2\right\}$ and $\Lambda^{-1}(\alpha-1, \alpha+1)=\left\{\left(\begin{array}{ll}\alpha & 1 \\ 1 & \alpha\end{array}\right)\right\}$, otherwise $\Lambda^{-1}\left(\left(\lambda_{1}, \lambda_{2}\right)\right)$ has two points $\left(\begin{array}{ll}x & 1 \\ 1 & y\end{array}\right)$ and $\left(\begin{array}{ll}y & 1 \\ 1 & x\end{array}\right)$. $\Lambda^{-1}\left(S_{2}^{\text {int }}\right)$ has two connected components where $b_{1}>b_{2}$ and where $b_{2}>b_{1}$. If one continues into the complex domain, $\Lambda^{-1}\left[\tilde{S}_{2} \backslash B\right]$ is connected.

Thus, our conjectures are true in the not quite trivial case $N=2$.

At first sight, it may seem surprising that $S_{N}$ is closed. After all, the eigenvalue image of all Jacobi matrices $\left\{\boldsymbol{\lambda} \in \mathrm{R}^{N} \mid \lambda_{1}<\lambda_{2}<\cdots<\lambda_{N}\right\}$ is open and not closed. The existence of strict inequalities is a reflection of the condition $a_{n}>0$. Once $a_{n} \equiv 1$, they disappear.

Theorem 7.4. $S_{N}$ is closed.

Proof. Let $\boldsymbol{\lambda}_{m} \in S_{N}$ and pick $\mathbf{b}_{m} \in \mathrm{R}^{N}$ so that $\Lambda\left(\mathbf{b}_{m}\right)=\boldsymbol{\lambda}_{m}$. Suppose $\boldsymbol{\lambda}_{m} \rightarrow \boldsymbol{\lambda}_{\infty} \in \mathrm{R}^{N}$ as $m \rightarrow \infty$. Let $H(\mathbf{b})$ be the $N \times N$ Schrödinger matrix with the components of $\mathbf{b}$ along the diagonal. Then

$$
|\Lambda(\mathbf{b})|^{2}=\operatorname{Tr}\left(H(\mathbf{b})^{2}\right)=2(N-1)+\|\mathbf{b}\|^{2},
$$

so $\left\{\mathbf{b}_{m}\right\}$ is a bounded subset of $\mathrm{R}^{N}$. Thus, we can find a subsequence $\left\{m_{p}\right\}$ such that $\mathbf{b}_{m_{p}} \rightarrow \mathbf{b}_{\infty}$ as $p \rightarrow \infty$. By continuity of $\Lambda, \Lambda\left(\mathbf{b}_{\infty}\right)=\boldsymbol{\lambda}_{\infty}$, that is, $\boldsymbol{\lambda}_{\infty} \in S_{n}$.

This theorem implies that if $\|\mathbf{b}\| \leq R$, then there is a minimum distance between eigenvalues. One might think there are global bounds on eigenvalue splittings (i.e., $N$ dependent but independent of $R$ ), but that is false if $N \geq 3$, as is seen by the following example motivated by tunneling considerations. Let $H(\beta)$ be the $N \times N$ Schrödinger matrix with $b_{1}=b_{N}=\beta$ and $b_{2}=\cdots=b_{N-1}=0$. Then for $\beta$ large, the two largest eigenvalues $E_{ \pm}(\beta)$ satisfy

$$
E_{ \pm}(\beta)=\beta \pm O\left(\beta^{-(N-2)}\right)
$$

and if $N \geq 3,\left|E_{+}(\beta)-E_{-}(\beta)\right| \rightarrow 0$ as $\beta \rightarrow \infty$.

An important open question is finding some kind of effective description of $S_{N}$. We note that if $\varphi_{+}=\left(\frac{1}{\sqrt{N}}, \ldots, \frac{1}{\sqrt{N}}\right)$ and $\varphi_{-}=\left(\frac{1}{\sqrt{N}},-\frac{1}{\sqrt{N}}, \frac{1}{\sqrt{N}}, \ldots, \frac{(-1)^{N+1}}{\sqrt{N}}\right)$, then $\left(\varphi_{+}, H \varphi_{+}\right)-$ $\left(\varphi_{-}, H \varphi_{-}\right)=4\left(1-\frac{1}{N}\right)$ so $\lambda_{N}-\lambda_{1} \geq 4\left(1-\frac{1}{N}\right)$.

The $N$ ! in our main conjecture comes from the following 
Theorem 7.5. For $\beta$ large, $\boldsymbol{\lambda}_{\beta}:=(\beta, 2 \beta, 3 \beta, \ldots, N \beta) \in S_{N}$ and $\Lambda^{-1}\left(\boldsymbol{\lambda}_{\beta}\right)$ has $N$ ! points. Proof. Consider the $N$ ! Hamiltonians

$$
H_{\pi}(\beta)=\beta\left(\begin{array}{ccc}
\pi(1) & & 0 \\
& \ddots & \\
0 & & \pi(N)
\end{array}\right)+\left(\begin{array}{cccc}
0 & 1 & & 0 \\
1 & \ddots & \ddots & \\
& \ddots & & 1 \\
0 & & 1 & 0
\end{array}\right),
$$

where $\pi$ is an arbitrary permutation on $\{1, \ldots, N\}$. Then $A(\beta)=\beta^{-1} H_{\pi}(\beta)$ at $\beta=0$ has $N$ eigenvalues $(1,2, \ldots, N)$ and it is easy to see that for $\beta$ small, the Jacobian of $\Lambda$ is invertible. It follows by the inverse function theorem that for $\beta$ large, there is a unique $\left.\tilde{H}_{\pi}(\beta)=H_{\pi}(\beta)+O(\beta)^{-1}\right)$ so that the eigenvalues of $\tilde{H}_{\pi}(\beta)$ are precisely $(\beta, 2 \beta, \ldots, N \beta)$.

A separate and easy argument shows that for $\beta$ large, any Schrödinger matrix with eigenvalues $(\beta, \ldots, N \beta)$ must have $b_{n}=\beta \pi(n)+O\left(\beta^{-1}\right)$ for some permutation $\pi$, and so must be one of the $\tilde{H}_{\pi}(\beta)$.

The evidence for the strong forms of the conjectures here is not overwhelming. We make them as much to stimulate further research as because we are certain they are true.

Acknowledgments. We would like to thank R. del Rio and G. Teschl for discussions and pertinent hints to the literature. F.G. is indebted to A. Kechris and C.W. Peck for a kind invitation to Caltech during the summer of 1996 where some of this work was done. The extraordinary hospitality and support by the Department of Mathematics at Caltech are gratefully acknowledged. B.S. would like to thank M. Ben-Artzi of the Hebrew University where some of this work was done.

\section{Appendix: Orthogonal Polynomials and the Inverse Problem}

Let $H$ be a Jacobi matrix on $\mathrm{N}$ or on $\{1, \ldots, N\}$, that is,

$$
\begin{aligned}
(H u)(n) & =a_{n} u(n+1)+b_{n} u(n)+a_{n-1} u(n-1), & & n \neq 1,(N) \\
& =a_{1} u(2)+b_{1} u(1), & & n=1 \\
& =b_{N} u(N)+a_{N-1} u(N-1), & & n=N
\end{aligned}
$$

where the $(N)$ refers to the finite matrix case. Here $a_{n}>0, b_{n} \in \mathrm{R}$, and we will suppose $a_{n}, b_{n}$ are bounded.

It will sometimes be useful to refer to a single sequence $c_{1}, c_{2}, \ldots:=b_{1}, a_{1}, b_{2}, a_{2}, \ldots$, that is,

$$
a_{n}=c_{2 n}, b_{n}=c_{2 n-1}, \quad n=1,2, \ldots
$$

Let $d \rho$ denote the spectral measure for the vector $\delta_{1}=(1,0, \ldots)$, that is,

$$
\left(\delta_{1}, e^{-i t H} \delta_{1}\right)=\int e^{-i t \lambda} d \rho(\lambda)
$$


In the finite case, $H$ has $N$ eigenvectors $\varphi_{1}, \ldots, \varphi_{N}$ with

$$
\left(\varphi_{j}, \varphi_{k}\right)=\delta_{j, k}, \quad H \varphi_{j}=\lambda_{j} \varphi_{j}
$$

and

$$
d \rho(\lambda)=\sum_{j=1}^{N}\left|\varphi_{j}(1)\right|^{2} \delta\left(\lambda-\lambda_{j}\right) d \lambda .
$$

Obviously, given $\left\{a_{n}\right\},\left\{b_{n}\right\}, d \rho$ is uniquely determined ( $H$ is bounded by the hypotheses on $a_{n}, b_{n}$ and so self-adjoint). It is an important fact that $d \rho$ determines $\left\{a_{n}\right\},\left\{b_{n}\right\}$, that any $d \rho$ of bounded support is allowed (in the finite case, any $N$-point measure is allowed; in the semi-infinite case, $\operatorname{supp}(d \rho)$ must be infinite).

Indeed, there is an elegant formalism for finding the $a$ 's and $b$ 's given $d \rho$. This formalism involves orthogonal polynomials. It has been discussed, for instance, in $[2,7,14-21,53$, 61]. We summarize it here for the reader's convenience and to fix notation.

We begin by analyzing the direct problem, that is, we suppose the $a$ 's and $b$ 's are given. Define functions $\{P(z, n)\}_{n=1}^{N}$ in the finite case and $\{P(z, n)\}_{n=1}^{\infty}$ in the $\mathrm{N}$ case by requiring

$$
\begin{aligned}
P(z, n+1) & =a_{n}^{-1}\left[\left(z-b_{n}\right) P(z, n)-a_{n-1} P(z, n-1)\right], \quad n \geq 2, \\
P(z, 1) & =1, P(z, 2)=a_{1}^{-1}\left(z-b_{1}\right) .
\end{aligned}
$$

The $P(z, n)$ for $z$ fixed and $n$ variable satisfy $(H-z) P(z)=0$ in the sense that if $\psi$ has finite support in the $\mathrm{N}$ case and if $\psi$ is supported on $[1,2, \ldots, N-1]$ in the finite case, then

$$
\sum_{n=1}^{N \text { or } \infty} P(z, n)((H-z) \psi)(n)=0 .
$$

Clearly, by induction $P(z, n)$ is a polynomial of degree $n-1$. Moreover,

$$
\tilde{P}(z, n)=a_{1} \ldots a_{n-1} P(z, n)
$$

are monic polynomials.

Proposition A.1. Define $P(H, n)$ using the functional calculus. Then

$$
P(H, n) \delta_{1}=\delta_{n}
$$

Proof. Clearly, (A.6) holds for $n=1$. Suppose it holds for $n=1, \ldots, n_{0}$. Then

$$
\begin{aligned}
\delta_{n+1} & =a_{n}^{-1}\left[H \delta_{n}-b_{n} \delta_{n}-a_{n-1} \delta_{n-1}\right] \\
& =a_{n}^{-1}\left[\left(H-b_{n}\right) P(H, n) \delta_{1}-a_{n-1} P(H, n-1) \delta_{1}\right] \\
& =P(H, n+1) \delta_{1}
\end{aligned}
$$

by the definition of $P$. Here we used the induction hypothesis in the second equality. 
Corollary A.2. The spectral measure for $\delta_{n}$ is $P(\lambda, n)^{2} d \rho(\lambda)$.

Corollary A.3.

$$
\int P(\lambda, m) P(\lambda, n) d \rho(\lambda)=\delta_{m, n}
$$

Proof. This just says that $\left(\delta_{m}, \delta_{n}\right)=\delta_{m, n}$.

Thus, the $P$ 's are the orthonormal polynomials defined by $d \rho$ via Gram-Schmidt on the functions $1, \lambda, \lambda^{2}, \ldots$ The $\tilde{P}$ are the more conventional orthogonal polynomials [2, 68] (orthogonal and monic rather than orthogonal and normalized). The defining relation of the $P$ 's,

$$
z P(z, n)=a_{n} P(z, n+1)+b_{n} P(z, n)+a_{n-1} P(z, n-1),
$$

or equivalently,

$$
z \tilde{P}(z, n)=\tilde{P}(z, n+1)+b_{n} \tilde{P}(z, n)+a_{n-1}^{2} \tilde{P}(z, n-1),
$$

are the standard three-term recursion relations for orthogonal polynomials.

Proposition A.4. The following determine each other

(i) $\{P(z, n)\}_{n=1}^{n_{0}+1}$

(ii) $\int \lambda^{j} d \rho(\lambda), \quad j=1,2, \ldots, 2 n_{0}$.

(iii) $\left\{c_{j}\right\}_{j=1}^{2 n_{0}}$.

Proposition A.5. The following determine each other

(i) $\{\tilde{P}(z, n)\}_{n=1}^{n_{0}+1}$

(ii) $\int \lambda^{j} d \rho(\lambda), \quad j=1,2, \ldots, 2 n_{0}-1$.

(iii) $\left\{c_{j}\right\}_{j=1}^{2 n_{0}-1}$.

Proofs. (A.8), (A.9) show that the claimed $c$ 's determine the $P$ 's (or $\tilde{P}$ 's) and vice-versa.

The $P$ 's and $\tilde{P}$ 's are a basis for polynomials up to $z^{n_{0}}$, so one can write $\left\{z^{j}\right\}_{j=0}^{n_{0}}$ in terms of the $P$ 's or $\tilde{P}$ 's. In the $P$ case, the orthogonality relations (A.7) then determine the integral $\int \lambda^{j} \lambda^{k} d \rho(\lambda)$ for $j, k=0,1, \ldots, n_{0}$ and so the stated moments. Conversely, by Gram-Schmidt, these moments determine the $P$ 's.

In the $\tilde{P}$ case, we argue as follows. The $\tilde{P}$ determine $a_{1}, \ldots, a_{n_{0}-1}$, and so by (A.5), $\{P(z, j)\}_{j=1}^{n_{0}}$. The orthonormality relations then determine the moments up to order $2 n_{0}-$ 2. The orthogonality relation $\int \lambda^{n_{0}-1} P\left(\lambda, n_{0}+1\right) d \rho(\lambda)$ determines the final $\int \lambda^{2 n_{0}-1} d \rho(\lambda)$ moment. Conversely, Gram-Schmidt and the moments determine the $\tilde{P}$ 's. Since we don't normalize $\tilde{P}\left(z, n_{0}+1\right)$, we don't need the $\int \lambda^{2 n_{0}} d \rho(\lambda)$ moment.

Theorem A.6. Every $N$-point probability measure $d \rho(\lambda)$ arises as the spectral measure of a unique $N \times N$ Jacobi matrix. Every probability measure of bounded and infinite support arises as the spectral measure of a unique Jacobi matrix on N.

Proof. Because a polynomial of degree $k$ has at most $k$ zeros, if $d \rho$ is supported on $N$ points, the monomials $\left\{\lambda^{n}\right\}_{n=0}^{N-1}$ are linearly independent in $L^{2}(d \rho)$. If $d \rho$ has infinite support, $\left\{\lambda^{n}\right\}_{n=0}^{\infty}$ are linearly independent in $L^{2}(d \rho)$. 
Thus given $d \rho$, we can use unnormalized Gram-Schmidt to construct monic polynomials $\{\tilde{P}(z, n)\}_{n=0}^{N-1}$ (with $N=\infty$ if $d \rho$ has infinite support). By construction, $\{\tilde{P}(z, n)\}_{n=0}^{p}$ is a basis for the polynomials of degree $p-1$. Thus,

$$
z \tilde{P}(z, n)=\tilde{P}(z, n+1)+\text { linear combination of } \tilde{P}(z, 1), \ldots, \tilde{P}(z, n) .
$$

The coefficient in front of $\tilde{P}(z, n+1)$ is one because $\tilde{P}(z, n)$ and $\tilde{P}(z, n+1)$ are monic. Since $z \tilde{P}(z, \ell)$ is a linear combination of $\{\tilde{P}(z, m)\}_{m=0}^{\ell+1}$, orthogonality implies $\int \tilde{P}(\lambda, k)[\lambda \tilde{P}(\lambda, \ell)] d \rho(\lambda)=$ 0 if $\ell+1<k$, and thus $z \tilde{P}(z, n)$ is orthogonal to $\{\tilde{P}(z, m)\}_{m=0}^{n-2}$, so

$$
z \tilde{P}(z, n)=\tilde{P}(z, n+1)+\beta_{n} \tilde{P}(z, n)+\alpha_{n} \tilde{P}(z, n-1),
$$

the standard three-term recursion relation.

Suppose $\operatorname{supp}(d \rho) \subseteq[-C, C]$. Then

$$
\beta_{n}=\int \lambda \tilde{P}(\lambda, n)^{2} d \rho(\lambda) / \int \tilde{P}(\lambda, n)^{2} d \rho(\lambda)
$$

and

$$
\alpha_{n}=\int \tilde{P}(z, n)^{2} d \rho(\lambda) / \tilde{P}(z, n-1)^{2} d \rho(\lambda)
$$

(since $\int \lambda \tilde{P}(\lambda, n) \tilde{P}(\lambda, n-1) d \rho(\lambda)=\int \tilde{P}(\lambda, n)[\lambda \tilde{P}(\lambda, n-1)] d \rho(\lambda)=\int \tilde{P}(\lambda, n)^{2} d \rho(\lambda)$ using $\left.z \tilde{P}(z, n-1)=\tilde{P}(z, n)+\beta_{n-1} \tilde{P}(z, n-1)+\alpha_{n-2} \tilde{P}(z, n-2)\right)$. Moreover, by (A.10), (A.12),

$$
\frac{\int \lambda^{2} \tilde{P}(\lambda, n)^{2} d \rho(\lambda)}{\int \tilde{P}(\lambda, n)^{2} d \rho(\lambda)} \geq \beta_{n}^{2}+\alpha_{n}^{2} \frac{1}{\alpha_{n}}=\beta_{n}^{2}+\alpha_{n} .
$$

Thus,

$$
0<\alpha_{n} \leq C^{2}, \quad\left|\beta_{n}\right| \leq C
$$

Comparing (A.10) and (A.9), we see that

$$
b_{n}=\beta_{n}, \quad a_{n}=\sqrt{\alpha_{n}}
$$

so that there is at most one Jacobi matrix that can have $d \rho$ as spectral measure (we recall the requirement $a_{n}>0$ ).

Given $d \rho$ of bounded support, define $a_{n}, b_{n}$ by (A.15). By (A.14), the Jacobi matrix is bounded. Let $d \tilde{\rho}$ be its spectral measure. By construction, the orthogonal polynomials for $d \rho$ and $d \tilde{\rho}$ are the same. Thus, their moments are the same by Propositions A.4 and A.5. But the moments uniquely determine a measure of finite support by Weierstrass' theorem on the density of polynomials in the continuous functions. Thus, $d \rho=d \tilde{\rho}$.

As an application of the formalism, we can translate Hochstadt's theorem [48] and proof into this language: 
Theorem A.7. (Hochstadt [48]) Given the eigenvalues $\lambda_{1}<\cdots<\lambda_{N}$ and the numbers $\left\{c_{j}\right\}_{j=1}^{N-1}$ of an $N \times N$ Jacobi matrix, there is at most one set of $\left\{c_{j}\right\}_{j=N}^{2 N-1}$ consistent with the data.

Proof $([48])$. Let $d \rho(\lambda)=\sum_{j=1}^{N} \alpha_{j} \delta\left(\lambda-\lambda_{j}\right) d \lambda$. We have to show the $\alpha_{j}$ are uniquely determined, since by Theorem A.6, they uniquely determine $\left\{c_{j}\right\}_{j=1}^{2 N-1}$. By Propositions A.4 and A.5, the $\left\{c_{j}\right\}_{j=1}^{N-1}$ determine the moments $m_{k}=\int \lambda^{k} d \rho(\lambda)$ for $k=1, \ldots, N-1$ and, of course, $m_{0}=1$. Thus we have

$$
\sum_{j=1}^{N} \lambda_{j}^{k} \alpha_{j}=m_{k}
$$

for $k=0,1, \ldots, N-1$. By the non-vanishing of van der Monde determinants,

$$
\operatorname{det}\left(\left(\lambda_{j}^{k}\right)_{0 \leq k \leq N-1,1 \leq j \leq N}\right)=\prod_{r<s}\left(\lambda_{r}-\lambda_{s}\right) \neq 0
$$

(A.16) has a unique solution. Thus, $\left\{c_{j}\right\}_{j=1}^{N-1}$ determines the moments $\left\{m_{k}\right\}_{k=0}^{N-1}$, the latter determines the $\left\{\alpha_{j}\right\}_{j=1}^{N}$, which in turn determines the $\left\{c_{j}\right\}_{j=1}^{2 N-1}$.

Remark. That there may be no matrix consistent with the data (see [23] for further discussion) comes from the fact that $\alpha_{j}$ 's determined by (A.16) may not be strictly positive.

\section{REFERENCES}

1. Z.S. Agranovich and V.A. Marchenko, The Inverse Problem of Scattering Theory, Gordon and Breach, New York, 1963.

2. N.I. Akhiezer, The Classical Moment Problem, Oliver and Boyd, Edinburgh, 1965.

3. A. Antony and M. Krishna, Almost periodicity of some Jacobi matrices, Proc. Indian Acad. Sci. 102 (1992), 175-188.

4. A. Antony and M. Krishna, Inverse spectral theory for Jacobi matrices and their almost periodicity, Proc. Indian Acad. Sci. 104 (1994), 777-818.

5. G.A. Baker and P. Graves-Morris, Padé Approximants, Parts I and II, Addison-Wesley, Reading, 1981.

6. D. Bättig, B. Grébert, J.-C. Guillot, and T. Kappeler, Fibration of the phase space of the periodic Toda lattice, J. Math. Pures Appl., to appear.

7. Ju.M. Berezanskii, Expansions in Eigenfunctions of Self-Adjoint Operators, Transl. Math. Mono. 17, Amer. Math. Soc., Providence, RI, 1968.

8. A.M. Bloch, H. Flaschka, and T. Ratiu, A convexity theorem for isospectral manifolds of Jacobi matrices in a compact Lie algebra, Duke Math. J. 61 (1990), 41-65. 
9. D. Boley and G.H. Golub, A survey of matrix inverse eigenvalue problems, Inverse Probl. 3 (1987), 595-622.

10. C. de Boor and G.H. Golub, The numerically stable reconstruction of a Jacobi matrix from spectral data, Lin. Algebra Appl. 21 (1978), 245-260.

11. G. Borg, Eine Umkehrung der Sturm-Liouvilleschen Eigenwertaufgabe, Acta Math. 78 (1946), 1-96.

12. G. Borg, Uniqueness theorems in the spectral theory of $y^{\prime \prime}+(\lambda-q(x)) y=0$, Proc. 11th Scandinavian Congress of Mathematicians, pp. 276-287, Johan Grundt Tanums Forlag, Oslo, 1952.

13. W. Bulla, F. Gesztesy, H. Holden, and G. Teschl, Algebro-geometric quasi-periodic finitegap solutions of the Toda and Kac-van Moerbeke hierarchies, Memoirs Amer. Math. Soc., to appear.

14. K.M. Case, On discrete inverse scattering problems, II, J. Math. Phys. 14 (1973), 916-920.

15. K.M. Case, Inverse problem in transport theory, Phys. Fluids 16 (1973), 1607-1611.

16. K.M. Case, The discrete inverse scattering problem in one dimension, J. Math. Phys. 15 (1974), 143-146.

17. K.M. Case, Scattering theory, orthogonal polynomials, and the transport equation, J. Math. Phys. 15 (1974), 974-983.

18. K.M. Case, Orthogonal polynomials from the viewpoint of scattering theory, J. Math. Phys. 15 (1974), 2166-2174.

19. K.M. Case, Orthogonal polynomials, II, J. Math. Phys. 16 (1975), 1435-1440.

20. K.M. Case and S.C. Chui, The discrete version of the Marchenko equations in the inverse scattering problem, J. Math. Phys. 14 (1973), 1643-1647.

21. K.M. Case and M. Kac, A discrete version of the inverse scattering problem, J. Math. Phys. 14 (1973), 594-603.

22. E. Date and S. Tanaka, Analogue of inverse scattering theory for the discrete Hill's equation and exact solutions for the periodic Toda lattice, Progr. Theoret. Phys. 55 (1976), 457-465.

23. P. Deift and T. Nanda, On the determination of a tridiagonal matrix from its spectrum and a submatrix, Lin. Algebra Appl. 60 (1984), 43-55.

24. P. Deift and E. Trubowitz, A continuum limit of matrix inverse problems, SIAM J. Math. Anal. 12 (1981), 799-818.

25. W.E. Ferguson, The construction of Jacobi and periodic Jacobi matrices with prescribed spectra, Math. Comp. 35 (1980), 1203-1220. 
26. H. Flaschka, On the Toda lattice, II, Progr. Theoret. Phys. 51 (1974), 703-716.

27. L. Fu and H. Hochstadt, Inverse theorems for Jacobi matrices, J. Math. Anal. Appl. 47 (1974), 162-168.

28. M.G. Gasymov and G.Sh. Guseinov, On inverse problems of spectral analysis for infinite Jacobi matrices in the limit-circle case, Sov. Math. Dokl. 40 (1990), 627-630.

29. I.M. Gel'fand and B.M. Levitan, On the determination of a differential equation from its special function, Izv. Akad. Nauk SSR. Ser. Mat. 15 (1951), 309-360 (Russian); English transl. in Amer. Math. Soc. Transl. Ser. 21 (1955), 253-304.

30. J.S. Geronimo and K.M. Case, Scattering theory and polynomials orthogonal on the unit circle, J. Math. Phys. 20 (1979), 299-310.

31. F. Gesztesy and H. Holden, Trace formulas and conservation laws for nonlinear evolution equations, Rev. Math. Phys. 6 (1994), 51-95.

32. F. Gesztesy and W. Renger, New classes of toda soliton solutions, Commun. Math. Phys. to appear.

33. F. Gesztesy and B. Simon, The xi function, Acta Math. 176 (1996), 49-71.

34. F. Gesztesy and B. Simon, Uniqueness theorems in inverse spectral theory for onedimensional Schrödinger operators, Trans. Amer. Math. Soc. 348 (1996), 349-373.

35. F. Gesztesy and B. Simon, Inverse spectral analysis with partial information on the potential, I. The case of an a.c. component in the spectrum, Helv. Phys. Acta, to appear.

36. F. Gesztesy and B. Simon, Inverse spectral analysis with partial information on the potential, II. The case of discrete spectrum, in preparation.

37. F. Gesztesy and G. Teschl, Commutation methods for Jacobi operators, J. Diff. Eqs. 128 (1996), 252-299.

38. F. Gesztesy, M. Krishna, and G. Teschl, On isospectral sets of Jacobi operators, Commun. Math. Phys., to appear.

39. G.M.L. Gladwell, On isospectral spring-mass systems, Inverse Probl. 11 (1995), 591602 .

40. W.B. Gragg and W.J. Harrod, The numerically stable reconstruction of Jacobi matrices from spectral data, Num. Math. 44 (1984), 317-335.

41. L.J. Gray and D.G. Wilson, Construction of a Jacobi matrix from spectral data, Lin. Algebra Appl. 14 (1976), 131-134.

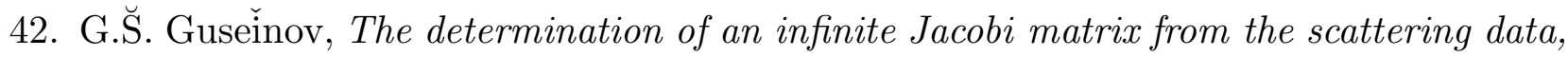
Sov. Math. Dokl. 17 (1976), 596-600. 


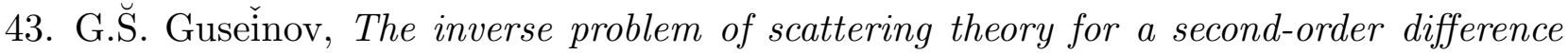
equation of the whole axis, Sov. Math. Dokl. 17 (1976), 1684-1688.

44. G.Sh. Guseinov, Determination of an infinite non-self-adjoint Jacobi matrix from its generalized spectral function, Math. Notes 23 (1978), 130-136.

45. O. Hald, Inverse eigenvalue problems for Jacobi matrices, Lin. Algebra Appl. 14 (1976), $63-85$.

46. H. Hochstadt, On some inverse problems in matrix theory, Arch. Math. 18 (1967), 201-207.

47. H. Hochstadt, On the construction of a Jacobi matrix from spectral data, Lin. Algebra Appl. 8 (1974), 435-446.

48. H. Hochstadt, On the construction of a Jacobi matrix from mixed given data, Lin. Algebra Appl. 28 (1979), 113-115.

49. H. Hochstadt and B. Lieberman, An inverse Sturm-Liouville problem with mixed given data, SIAM J. Appl. Math. 34 (1978), 676-680.

50. M. Kac and P. van Moerbeke, On some periodic Toda lattices, Proc. Nat. Acad. Sci. USA 72 (1975), 1627-1629.

51. M. Kac and P. van Moerbeke, A complete solution of the periodic Toda problem, Proc. Nat. Acad. Sci. USA 72 (1975), 2879-2880.

52. M. Kac and P. van Moerbeke, On an explicitly soluble system of nonlinear differential equations related to certain Toda lattices, Adv. Math. 16 (1975), 160-169.

53. H.J. Landau, The classical moment problem: Hilbertian proofs, J. Funct. Anal. 38 (1980), 255-272.

54. N. Levinson, The inverse Sturm-Liouville problem, Mat. Tidskr. B (1949), 25-30.

55. B. Levitan, On the determination of a Sturm-Liouville equation by two spectra, Amer. Math. Soc. Transl. 68 (1968), 1-20.

56. B. Levitan, Inverse Sturm-Liouville Problems, VNU Science Press, Utrecht, 1987.

57. B.M. Levitan and M.G. Gasymov, Determination of a differential equation by two of its spectra, Russ. Math. Surv. 19:2 (1964),1-63.

58. B. Levitan and I. Sargsjan, Sturm-Liouville and Dirac Operators, Kluwer, Dordrecht, 1991.

59. V.A. Marchenko, Some questions in the theory of one-dimensional linear differential operators of the second order, I, Trudy Moskov. Mat. Obŭč 1 (1952), 327-420 (Russian); English transl. in Amer. Math. Soc. Transl. (2)101 (1973), 1-104.

60. V. Marchenko, Sturm-Liouville Operators and Applications, Birkhäuser, Basel, 1986. 
61. D. Masson and J. Repka, Spectral theory of Jacobi matrices in $\ell^{2}(\mathrm{z})$ and the $S U[1,1]$ Lie algebra, SIAM J. Math. Anal. 22 (1991), 1131-1145.

62. P. van Moerbeke, The spectrum of Jacobi matrices, Invent. math. 37 (1976), 45-81.

63. P. van Moerbeke and D. Mumford, The spectrum of difference operators and algebraic curves, Acta Math. 143 (1979), 93-154.

64. J. Pöschel and E. Trubowitz, Inverse Scattering Theory, Academic Press, Boston, 1987.

65. B. Simon, Spectral analysis of rank one perturbations and applications, CRM Lecture Notes Vol. 8 (J. Feldman, R. Froese, L. Rosen, eds.), pp. 109-149, Amer. Math. Soc., Providence, RI, 1995.

66. M.L. Sodin and P.M. Yuditskǐ, Infinite-zone Jacobi matrices with pseudo-exten-dible Weyl functions and homogeneous spectrum, Russ. Acad. Sci. Dokl. Math. 49 (1994), $364-368$.

67. M.L. Sodin and P.M. Yuditskii, Infinite-dimensional Jacobi inversion problem, almostperiodic Jacobi matrices with homogeneous spectrum, and Hardy classes of characterautomorphic functions, preprint, 1994.

68. G. Szego, Orthogonal Polynomials, Amer. Math. Soc. Colloq. Publ. 23, New York, 1939 .

69. G. Teschl, Trace formulas and inverse spectral theory for Jacobi operators, preprint, 1996.

70. C. Tomei, The topology of isospectral manifolds of tridiagonal matrices, Duke Math. J. 51 (1984), 981-996.

71. B.N. Zakhar'iev, V.N. Mel'nikov, B.V. Rudyak, and A.A. Suz'ko, Inverse scattering problem (finite-difference approach), Sov. J. Part. Nucl. 8 (1977), 120-137. 